\title{
Continuing our work: transplant surgery and surgical oncology in a tertiary referral COVID-19 center
}

\author{
Giammauro Berardi ${ }^{1}$ (D) Marco Colasanti ${ }^{1}$. Giovanni Battista Levi Sandri ${ }^{1}$. Celeste Del Basso ${ }^{1}$. Stefano Ferretti ${ }^{1}$. \\ Andrea Laurenzi ${ }^{1}$ - Nicola Guglielmo ${ }^{1}$. Roberto Luca Meniconi ${ }^{1} \cdot$ Mario Antonini ${ }^{2}$. Gianpiero $D^{\prime}$ Offizi $^{3}$. \\ Giuseppe Maria Ettorre ${ }^{1}$
}

Received: 25 April 2020 / Accepted: 28 May 2020 / Published online: 4 June 2020

(C) Italian Society of Surgery (SIC) 2020

\begin{abstract}
COVID-19 is rapidly spreading worldwide. Healthcare systems are struggling to properly allocate resources while ensuring cure for diseases outside of the infection. The aim of this study was to demonstrate how surgical activity was affected by the virus outbreak and show the changes in practice in a tertiary referral COVID-19 center. The official bulletins of the Italian National Institute for the Infectious Diseases "L. Spallanzani" were reviewed to retrieve the number of daily COVID-19 patients. Records of consecutive oncological and transplant procedures performed during the outbreak were reviewed. Patients with a high probability of postoperative intensive care unit (ICU) admission were considered as high risk and defined by an ASA score $\geq$ III and/or a Charlson Comorbidity Index (CCI) $\geq 6$ and/or a Revised Cardiac Risk Index for Preoperative Risk $(\mathrm{RCRI}) \geq 3.72$ patients were operated, including $12(16.6 \%)$ liver and kidney transplantations. Patients had few comorbidities (26.3\%), low ASA score $(1.9 \pm 0.5)$, CCI $(3.7 \pm 1.3)$, and RCRI $(1.2 \pm 0.6)$ and had overall a low risk of postoperative ICU admission. Few patients had liver cirrhosis (12.5\%) or received preoperative systemic therapy (16.6\%). $36(50 \%)$ high-risk surgical procedures were performed, including major hepatectomies, pancreaticoduodenectomies, total gastrectomies, multivisceral resections, and transplantations. Despite this, only 15 patients (20.8\%) were admitted to the ICU. Only oncologic cases and transplantations were performed during the COVID-19 outbreak. Careful selection of patients allowed to perform major cancer surgeries and transplantations without further stressing hospital resources, meanwhile minimizing collateral damage to patients.
\end{abstract}

Keywords COVID-19 - General surgery $\cdot$ Surgical oncology

\section{Introduction}

Since December 2019, the world is struggling against Coronavirus Disease 2019 (COVID-19) and in the past months, the life of more than one-third of the planet's population

Giammauro Berardi

gberardi1@gmail.com

1 Department of General Surgery and Liver Transplantation Service, San Camillo Forlanini, Italian National Institute for the Infectious Diseases “L. Spallanzani”, Via Giacomo Folchi 6A, Rome, Italy

2 Department of Intensive Care and Anesthesiology, San Camillo Forlanini, Italian National Institute for the Infectious Diseases “L. Spallanzani”, Rome, Italy

3 Department of Infectious Diseases, Italian National Institute for the Infectious Diseases “L. Spallanzani”, Rome, Italy has radically changed [1]. Severe Acute Respiratory Syndrome Coronavirus 2 (SARS-CoV-2) has shown to be a very contagious virus potentially causing complicated atypical pneumonia and associated with significant mortality [2]. Despite initially confined to Hubei province, China and Asia, COVID-19 promptly spread to western countries, and in March 11, 2020, the World Health Organization (WHO) declared a public health emergency of international concern [3]. By early April, we are counting over 1,500,000 cases and 88,000 deaths worldwide, with 209 countries involved over 4 continents [4]. Italy was one of the first western countries diagnosing COVID-19 patients in late January and was certainly the one suffering the most by the sudden outbreak of the disease. Indeed, SARS-CoV-2 rapidly spread throughout the country by the end of February, challenging the healthcare system toward its collapse, increasing the mortality rate and raising the question concerning the need 
for special measures to contain the crisis. As a matter of fact, by mid-March the Italian cases of COVID- 19 were already 20,000 with almost 2000 patients requiring mechanical ventilation in the intensive care unit (ICU) [5]. Consequently, the Italian Government announced extraordinary measures to contain the spread of the pandemic, by means of small confinements in northern Italy, reaching a nationwide lockdown that is still limiting the population [6].

COVID-19 outbreak seriously stressed the healthcare system worldwide, with the urgent need of extra ICU beds, dedicated hospital paths, personnel training, and infection control measures [7, 8]. Care of diseases outside the COVID-19 has rapidly changed, and healthcare providers are currently adapting to maximize and properly allocate resources [9]. Surgical activity has shifted to pursue only emergency and elective cancer cases as described in different specialties [10-13]. Furthermore, the big picture and the full impact is still unknown as its effects on economy, hospital infrastructure, healthcare strategy, and prognosis of oncologic patients [14]. Indeed, people with cancer might suffer the most by this worldwide outbreak as the access to surgery is limited by the significant reduction in available resources, eventually influencing the decision making and the therapeutic allocation of patients [15].

The Italian experience anticipated what the world is currently facing, and the measures for containment, the healthcare adaptations, and the changes in hospital practice have been already tested and are somehow established in our country where the peak of the infection seems to be reached and overcome [5]. As the Italian National Institute for the Infectious Diseases, we have hospitalized the first Italian COVID-19 patients and since then, our general surgery department had to face this reality [16]. The aim of this study was to demonstrate how our surgical activity was affected by the COVID-19 outbreak, what adaptations were made and how our practice changed, eventually sharing the lessons learned as anticipators in a health issue of international concern.

\section{Materials and methods}

On January 31, two COVID-19-positive individuals were hospitalized at the Italian National Institute for the Infectious Diseases "L. Spallanzani" of Rome. In this hospital, four departments and five daily outpatient clinics are dedicated to the diagnosis and treatment of various infectious diseases. Health emergencies, such as SARS in 2003 and Ebola in 2014, have been nationally referred and managed at our hospital previously $[17,18]$. In the same institution, our general surgery department operates in two daily operative rooms (OR) more than 900 cases per year, including liver, kidney, and pancreas transplantations. The ICU of the hospital admits both medical and surgical patients in a 9-bed department.

The records and official bulletins of our hospital were reviewed to retrieve the number of daily COVID-19 patients hospitalized, those requiring ICU admission and those discharged home. Furthermore, all the records of consecutive surgical procedures performed during the COVID-19 outbreak were reviewed, and the following data were extracted: age, gender, disease, comorbidities, neoadjuvant therapy, cirrhosis, previous surgery, type of surgical procedure, type of approach (open vs. laparoscopic), conversion, operative time, blood loss, and admission to the ICU. The search was limited between the nationwide lockdown (March 9, 2020) and April 24, 2020.

Informed consent was obtained from each patient, and every oncologic case was discussed in a multidisciplinary meeting involving surgeons, medical oncologists, gastroenterologists, radiologists, and pathologists.

\section{Definitions}

Comorbidities were graded using the American Society of Anesthesiology (ASA) score, the Charlson Comorbidity index (CCI), and the Revised Cardiac Risk Index for Preoperative Risk (RCRI). Patients with a high probability of postoperative ICU admission were considered as high risk and defined by an ASA score $\geq \mathrm{III}$ and/or a CCI $\geq 6$ and/or a RCRI $\geq 3$ as previously validated [19-21]. Major hepatectomies were defined as the resections of three liver segments or more [22]. Pancreatic resections, total gastrectomies, major hepatectomies, and multivisceral resections as well as liver and kidney transplantations were considered as the high-risk surgical procedures because of the increased likelihood of postoperative ICU admission.

\section{Statistical analysis}

Distribution of variables was assessed using Kolmogorov-Smirnov and Shapiro-Wilk tests.

Data are expressed as the mean \pm standard deviation for parametric continuous data and as median and interquartile ranges for non-parametric distribution. Categorical data were expressed as number and percentages. Categorical data are expressed as number and percentages. Chi-squared or Fisher exact test with Yates correction when appropriate was used to compare differences in categorical variables. Unpaired student's t-test was used to compare differences in continuous parametric variables and the Mann-Whitney test for continuous non-parametric variables. Statistical analysis was performed with SPSS software (version 20.0. Armonk, NY, IBM Corp) for MacOsX. A $p$ value of $<0.05$ was considered statistically significant. 


\section{Results}

\section{Timeline of events since the hospitalization of the first COVID-19 patients in Italy}

On January 31, 2020 (Day 0), the first two COVID-19-positive patients in Italy were admitted to the department of infectious diseases of our hospital with mild fever and atypical pneumonia requiring no invasive treatment. On February 4 (Day 4), due to the worsening clinical conditions, mechanical ventilation was needed, and the two patients were admitted to the ICU. Three days later (Day 7), a third patient with positive nasopharyngeal swab was admitted and monitored at the department of infectious diseases. From Day 4 to Day 30 (March 1, 2020), diagnostic nasopharyngeal swabs for symptomatic patients were implemented at our institution as part of a regional referral policy; however, no further patients tested positive to SARS-CoV-2, picturing a stable phase of the epidemic. On March 2 (Day 31), two individuals had a positive swab and were hospitalized; since then, an exponential growth of COVID-19 cases admitted to our hospital was registered as part of a national trend. Sixty-five hospitalized cases were reached on Day 34, nine of which required mechanical ventilation, therefore saturating the capacity of the ICU. On the evening of March 8, the Italian government announced the national lockdown, and the following day (Day 38), the first Institutional guidelines on extraordinary COVID-19 measures were released by our hospital (Fig. 1): (1) All healthcare workers were required to enhance hygienic measures and wear surgical masks and gloves within the hospital building. (2) Construction works in the ICU started with the aim of increasing the number of beds from 9 to 25. (3) One of our daily general surgery operating rooms was closed to reallocate anesthesiologists and nurses to the management of COVID-19 cases. (4) The OR personnel was trained on how to wear and un-wear special equipment and how to deal with contagious patients. (5) Our surgical department was asked to re-consider and re-work the OR planning to perform only oncological cases and transplantations; outpatient clinics were significantly reduced. (6) Questionnaires on possible infection or contacts with COVID-19 cases were administered to all patients admitted and scheduled for surgery; nasopharyngeal swabs were performed in suspicious cases. (7) All gatherings, including our multidisciplinary meetings, were forbidden and reworked on a webinar platform.

On March 23 (Day 52), further Institutional guidelines were released: (1) Relatives were forbidden to visit patients before and after surgery. (2) Nasopharyngeal swabs were administered to all patients admitted and scheduled for surgery and isolation was maintained until test's response. As a result, the first COVID-19 patient of our surgical ward was identified on Day 53: the man had a Klatskin tumor and was scheduled to receive a right hepatectomy with hepaticojejunostomy. Unfortunately, he was shifted to percutaneous transhepatic biliary drainage and transferred to the infectious disease department.

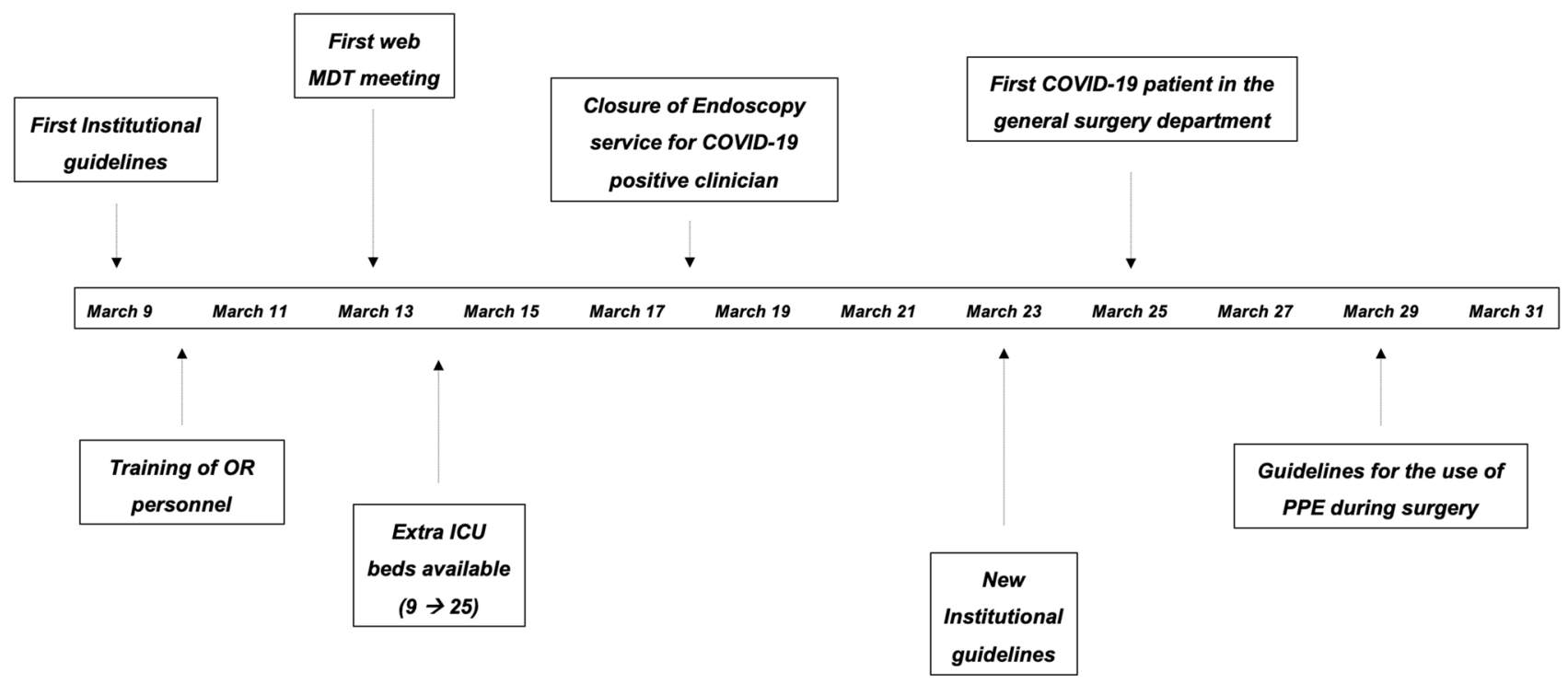

OR, Operative room. MDT, Multidisciplinary team. ICU, Intensive Care Unit. COVID-19, Coronavirus disease-19. PPE, Personal Protective Equipment.

Fig. 1 Timeline of events in our hospital since Italian national lockdown 


\section{Surgical practice during COVID-19}

Seventy-two patients (47 males and 25 females) with a median age of 64 (53-74) were operated at our surgical department in the study period (Table 1). Most patients had colorectal cancer $(22.2 \%)$ and colorectal liver metastases $(18.0 \%)$, followed by cholangiocarcinoma (11.1\%) and pancreatic ductal adenocarcinoma $(8.3 \%)$. Twelve patients $(16.6 \%)$ had liver or kidney disease requiring transplantation and no patients died while on the waiting list. Compared to the same period of time in 2019, fewer procedures were performed in 2020 (72 in 2020 vs. 115 in 2019); furthermore, a statistical significant difference

Table 1 Characteristics of patients operated during the COVID-19 outbreak (March 9-April 24, 2020)

\begin{tabular}{ll}
\hline & $n=72$ \\
\hline Age (years) & $64(53-74)$ \\
Gender F/M & $25 / 47$ \\
Comorbidities & $19(26.3 \%)$ \\
ASA score & $1.9 \pm 0.5$ \\
Charlson Comorbidity Index & $3.7 \pm 1.3$ \\
Revised Cardiac Risk Index for Preoperative risk & $1.2 \pm 0.6$ \\
Previous surgery & $21(30.5 \%)$ \\
Cirrhosis & $9(12.5 \%)$ \\
Neoadjuvant systemic therapy & $12(16.6 \%)$ \\
High-risk patients & $16(22.2 \%)$ \\
Diagnosis & \\
Colorectal cancer & $16(22.2 \%)$ \\
Cholangiocarcinoma & $8(11.1 \%)$ \\
Colorectal liver metastases & $13(18.0 \%)$ \\
Hepatocellular carcinoma & $3(4.1 \%)$ \\
Pancreatic ductal adenocarcinoma & $6(8.3 \%)$ \\
Gastric cancer & $3(4.1 \%)$ \\
Renal clear cell carcinoma & $4(3.6 \%)$ \\
Sarcoma & $2(5.5 \%)$ \\
Adrenal cancer & $1(1.3 \%)$ \\
Gastrointestinal stromal tumor & $2(5.5 \%)$ \\
Other malignancy & \\
HCV related liver cirrhosis & $2(5.5 \%)$ \\
HIV related liver cirrhosis & $2(5.5 \%)$ \\
Alcohol related liver cirrhosis & $1(1.3 \%)$ \\
NAFLD & $1(1.3 \%)$ \\
Autoimmune hepatitis & $1(1.3 \%)$ \\
Chronic kidney failure & $1(1.3 \%)$ \\
\hline & $6(8.3 \%)$ \\
\hline
\end{tabular}

Data expressed as median and interquartile ranges or absolute count and percentages

ASA American Society of Anesthesiology, $H C V$ hepatitis $\mathrm{C}$ virus, $H I V$ human immunodeficiency virus, NAFLD non-alcoholic fatty liver disease

${ }^{\mathrm{a}}$ One liver cistoadenocarcinoma, one suspicious malignant abdominal lymph node in terms of type of operations was found between years (23.4\% benign, $73.9 \%$ malignant, and $2.6 \%$ transplantations in 2019 vs. $0 \%$ benign, $86.4 \%$ malignant, and $16.6 \%$ transplantations in 2020; $p<0.001$ ).

Overall, patients operated during the COVID-19 outbreak had few comorbidities (26.3\%), low ASA score (1.9 \pm 0.5$)$, CCI ( $3.7 \pm 1.3)$, and RCRI $(1.2 \pm 0.6)$, being overall at lower risk of postoperative ICU admission (Table 1). Furthermore, few patients had liver cirrhosis $(12.5 \%)$ or received preoperative systemic therapy (16.6\%). Type of surgical procedures and operative details are depicted in Table 2. We have performed 36 $(50.0 \%)$ high-risk operations in the study period of which $6(8.3 \%)$ were liver and $6(8.3 \%)$ were kidney transplantations. Despite this, few patients were admitted to the ICU (20.8\%). One patient (1.3\%) died during the study period (one hyperacute allograft dysfunction following liver transplantation for autoimmune hepatitis). Morbidity rate was $19.4 \%$ and five patients $(6.9 \%)$ developed a major grade complication according to Clavien-Dindo.

Table 2 Type of surgical procedures and operative details of patients operated during the COVID-19 outbreak (March 9-April 24, 2020)

\begin{tabular}{|c|c|}
\hline & $n=72$ \\
\hline High-risk surgical procedures & $36(50.0 \%)$ \\
\hline \multicolumn{2}{|l|}{ Type of surgical procedure } \\
\hline Colorectal resection $^{\mathrm{a}}$ & $15(20.8 \%)$ \\
\hline Major hepatectomy w/o hepaticojejunostomy & $14(19.4 \%)$ \\
\hline Minor hepatectomy & $11(15.2 \%)$ \\
\hline Pancreaticoduodenectomy & $6(8.3 \%)$ \\
\hline Total gastrectomy & $1(1.3 \%)$ \\
\hline Distal gastrectomy & $2(2.7 \%)$ \\
\hline Nephrectomy & $2(2.7 \%)$ \\
\hline Multivisceral resection & $2(2.7 \%)$ \\
\hline Other malignant procedures ${ }^{\mathrm{b}}$ & $7(9.7 \%)$ \\
\hline Orthotopic liver transplantation $^{c}$ & $6(8.3 \%)$ \\
\hline Kidney transplantation & $6(8.3 \%)$ \\
\hline Laparoscopic approach & $26(36.1 \%)$ \\
\hline Conversion & $0(0 \%)$ \\
\hline Blood loss (mL) & $400(100-600)$ \\
\hline Operative time (min) & $390(200-400)$ \\
\hline Patients admitted to the ICU & $15(20.8 \%)$ \\
\hline
\end{tabular}

$I C U$ intensive care unit

${ }^{\text {a }}$ Four right colectomies, nine left colectomies, and two anterior rectal resections

${ }^{\mathrm{b}}$ Two gastric wedge resections, one nephrectomy with removal of inferior vena cava a right atrium tumor thrombus, one partial nephrectomy, one adrenalectomy, one transanal endoscopic mucosectomy, and one abdominal lymph node biopsy

${ }^{\mathrm{c}}$ MELD score of patients at the time of liver transplantation: 28 (2333) 


\section{Changes in surgical practice during the two phases of COVID-19 outbreak}

As mentioned above, during the first period (January 31-March 8, 2020), COVID-19 hospitalizations were stable at our hospital, with two cases admitted to the ICU and one monitored at the department of infectious diseases. In the second period (March 9-April 24, 2020), an exponential growth in number of admissions was registered, with a median of 10 (4-21) positive cases per day (Fig. 2). At the same time, a median of $3(2-4)$ cases per day were operated by our surgical team in the first period, while fewer cases were operated in the second (median of 2 (1-2) per day; $p=0.001)$. Thirty-nine (41.9\%) benign conditions, 48 (51.6\%) oncological patients, and $6(6.5 \%)$ transplantations were performed in the first period, while only malignant cases and transplantations $(n=72,100 \%)$ were performed in the second.

Considering only the transplantations and the operations performed for cancer, patients in the second period had fewer comorbidities, lower ASA score, CCI, and RCRI, being overall at lower risk of postoperative ICU admission (Table 3). Furthermore, fewer patients had previous surgery (30.5\% vs. $57.4 \% ; p<0.001)$ and/or received preoperative systemic therapy $(16.6 \%$ vs. $48.1 \%$; $p<0.001)$. The number of high-risk operations was higher in the second phase of the pandemic $(27.8 \%$ vs. $50.0 \% ; p=0.01)$. Despite this, fewer patients were admitted to the ICU in period 2 and this was statistically significant ( $37.0 \%$ in period 1 vs. $20.8 \%$ in period $2 ; p=0.04)$.

\section{Discussion}

In this study, we have shown how the recent COVID-19 outbreak affected our department of surgery and transplantation. Fewer surgeries were performed and these were only for cancer and for urgent liver or kidney transplantations. Notwithstanding, we have maintained our standard practice by enhancing selection of patients, meanwhile allowing for the re-allocation of the hospital resources.

SARS-CoV-2 virus infection has dramatically changed the world recently. More than two hundred countries all over the world had to undertake extraordinary measures to contain the epidemic and reduce possible side effects on healthcare and economy [1]. Indeed, many eastern and western governments followed the example of China and are currently in lockdown, with few exceptions. A deep impact was expected on the healthcare systems all over the world: re-allocation of resources was urgently required in an effort to contain the devastating consequences of the pandemic. As a domino effect, patients with other medical conditions, such as cardiovascular diseases, cancer, and chronic conditions, suffered and are still suffering as a collateral damage [15].

In response to the rapid increase of COVID-19 cases, Institutional guidelines called for the re-arrangement of the



Fig. 2 Number of COVID-19 patients per day and general surgery cases since the hospitalization of the first COVID-19 patients 
Table 3 Characteristics of patients operated for malignancy according to the two phases of the COVID-19 outbreak

\begin{tabular}{|c|c|c|c|}
\hline & Jan 31-Mar $82020, n=54$ & $\begin{array}{l}\text { Mar 9-April } 24 \\
2020, n=72\end{array}$ & $p$ \\
\hline Age (years) & $64(53-74)$ & $64(53-77)$ & 0.38 \\
\hline Gender F/M & $22 / 26$ & $25 / 47$ & 0.22 \\
\hline Comorbidities & $29(53.7 \%)$ & $6(21.4 \%)$ & 0.001 \\
\hline ASA score & $2.3 \pm 0.5$ & $1.9 \pm 0.5$ & 0.004 \\
\hline Charlson Comorbidity Index & $5.1 \pm 1.2$ & $3.7 \pm 1.3$ & $<0.001$ \\
\hline Revised Cardiac Risk Index for preoperative risk & $2.2 \pm 0.7$ & $1.2 \pm 0.6$ & $<0.001$ \\
\hline Previous surgery & $31(57.4 \%)$ & $21(30.5 \%)$ & $<0.001$ \\
\hline Cirrhosis & $9(16.7 \%)$ & $9(12.5 \%)$ & 0.34 \\
\hline Neoadjuvant systemic therapy & $26(48.1 \%)$ & $12(16.6 \%)$ & $<0.001$ \\
\hline High-risk patients & $25(46.3 \%)$ & $16(22.2 \%)$ & 0.001 \\
\hline High-risk surgical procedures & $15(27.8 \%)$ & $36(50.0 \%)$ & 0.01 \\
\hline Type of surgical procedure & & & 0.89 \\
\hline Colorectal resection & $7(12.9 \%)$ & $15(20.8 \%)$ & \\
\hline Major hepatectomy w/o hepaticojejunostomy & $7(12.9 \%)$ & $14(19.4 \%)$ & \\
\hline Minor hepatectomy & $12(22.2 \%)$ & $11(15.2 \%)$ & \\
\hline Pancreaticoduodenectomy & $4(7.4 \%)$ & $6(8.3 \%)$ & \\
\hline Distal pancreatectomy & $1(1.8 \%)$ & $0(0 \%)$ & \\
\hline Esophagectomy & $1(1.8 \%)$ & $0(0 \%)$ & \\
\hline Total gastrectomy & $1(1.8 \%)$ & $1(1.3 \%)$ & \\
\hline Distal gastrectomy & $0(0 \%)$ & $2(2.7 \%)$ & \\
\hline Nephrectomy & $3(5.5 \%)$ & $2(2.7 \%)$ & \\
\hline Multivisceral resection & $1(1.8 \%)$ & $2(2.7 \%)$ & \\
\hline Other malignant procedures & $11(20.3 \%)$ & $7(9.7 \%)$ & \\
\hline Orthotopic liver transplantation & $3(5.5 \%)$ & $6(8.3 \%)$ & \\
\hline Kidney transplantation & $3(5.5 \%)$ & $6(8.3 \%)$ & \\
\hline Laparoscopic approach & $17(31.4 \%)$ & $26(36.1 \%)$ & 0.93 \\
\hline Conversion & $1(1.8 \%)$ & $0(0 \%)$ & 0.63 \\
\hline Blood loss (mL) & $165(62-450)$ & $400(100-600)$ & 0.77 \\
\hline Operative time (min) & $381(226-488)$ & $390(200-400)$ & 0.12 \\
\hline Patients admitted to the ICU & $20(37 \%)$ & $15(20.8 \%)$ & 0.04 \\
\hline
\end{tabular}

Data expressed as median and interquartile ranges or absolute count and percentages

Bold reflects statistically significant results

ASA American Society of Anesthesiology, ICU intensive care unit hospital, shifting both infrastructures and personnel toward the containment of the infection and the management of SARS-CoV-2-positive patients. Anesthesiologists and nurses were re-allocated, and dedicated teams for both COVID-19 patients and surgical practice were created. Our department suffered the sudden closure of one daily OR and the significant reduction in the possibility to use ICU beds after surgery, as these were day by day used for the management of COVID-19 patients. As surgeons, we had to completely re-consider our roles and our surgical activity, reckoning with the limited resources. We had to face a healthcare emergency with uncertain future course and adapt accordingly. Surgeries for benign conditions were stopped and the waiting list for cancer was handled and reworked entirely. The most important limitation was the hampered access to the ICU as many patients require intensive monitoring after surgery for cancer, especially when major procedures are needed. Furthermore, we had to deal with the fact that transplantations normally require intensive care management in the postoperative. We were advised to limit the number of surgeries requiring ICU admission as much as possible, to avoid the saturation of resources and failure of the system. As an institutional policy, our transplant center remained opened and we decided to continue with our standard surgical oncology activity, improving selection of patients to limit the need for postoperative intensive care management. Waiting list for liver and kidney transplantations was maintained respecting the same inclusion and exclusion criteria and using the same prioritization. Transplantations were still admitted to the ICU for monitoring through a dedicated 
pathway with dedicated personnel, despite limiting their length of stay and discharging them to the surgical ward as soon as possible. Notably, we have performed standard numbers of the high-risk surgical procedures, selecting more surgically fit individuals. Furthermore, patients were less commonly having preoperative neoadjuvant chemotherapy and/or previous surgery, significantly lowering intraoperative risks. All the transplantations performed were admitted to the ICU, while only three oncologic patients required intensive care management after surgery: these latter were patients with significant comorbidities that were scheduled for the high-risk surgeries (two pancreaticoduodenectomies for adenocarcinoma and one right hepatectomy for hepatocellular carcinoma). Our policy was pursued in an effort to prioritize patients by cancer prognosis. Indeed, malignancies requiring high-risk surgical interventions (i.e., cholangiocarcinoma, liver metastasis, pancreatic adenocarcinoma, and sarcoma) are generally those with the worst prognosis and more likely to suffer by a delay in treatment [23]. A different approach would have been to rework the waiting list for cancer prioritizing less-invasive procedures (i.e., colorectal resections) with no strict selection of candidates in order to cope with the limited resources [24]. The main issue here is that both strategies will generate a gap in cure that has to be considered in the near future when the COVID-19 crisis will be over. Surgical delay should not affect oncological prognosis, in order to minimize future repercussions of this international health crisis [23]. The alternatives to surgery and their efficacy should be reviewed and discussed for each type of cancer and case by case, in an effort to minimize the waiting list and the loss of patients due to cancer progression $[25,26]$. In some cases, systemic treatment could be prolonged or adapted, while in others the window of cure might be missed; sometimes surgery can be postponed, while some other patients need surgical treatment to stay on their pathway $[27,28]$. In this setting, multidisciplinary meetings should be reworked to consider patients with cancer in the COVID-19 era, in which resources and future are uncertain. Balancing the prognosis of cancer with the potential alternatives and the characteristics of patients might eventually lead to improve the treatment allocation exploiting resources and maintaining oncologic principles [29]. Finally, an "hub and spoke" system based on a regional policy should be encouraged in the near future to allow for immediate referral and faster access to treatments, eventually improving quality of care.

Patients with cancer are paying the price at different levels, not only because of the limited access to cure but also because of the delay in diagnosis [30]. Indeed, the significant reduction of outpatient clinics and the limitation in imaging modalities and/or endoscopies might hamper a rapid diagnosis and a prompt referral. As a further issue, it has already been shown that cancer patients are more susceptible to COVID-19 infections: 1\% of COVID-19 patients in China had a history of cancer as compared to $0.29 \%$ among normal Chinese population [2]. These patients were also having more severe infections and most commonly required intubation and invasive ventilation. One lesson that we have learned in our hospital is that the diagnostic modalities for COVID-19 are important not only to contain the infection and avoid the spread of the disease but also to ensure safe treatment of patients. Questionnaires are useless in our opinion, as we are at the point that this pandemic cannot be detected by recalling contacts or possible exposure to the infection [31]. Indeed, once we have shifted to nasopharyngeal swabs for all patients admitted to our department, we had our first SARS-CoV-2-positive surgical candidate, this patient earlier tested negative to the questionnaire. On one hand, this highlights the importance of swabs for all surgical candidates to contain the infection among patients and healthcare workers; on the other hand, it sheds the light on the need for further evidence concerning the management of positive COVID-19 patients with cancer. Indeed, our patient with cholangiocarcinoma was shifted to non-surgical treatment: our team was unprepared at that time as no recommendations were available and issues regarding potential complications and transmission were raised. Recently, Lei et al. have demonstrated that 34 positive asymptomatic COVID-19 patients undergoing surgery had a $20 \%$ mortality rate, and all developed atypical pneumonias. Furthermore, it is very important to stress that our decision unfortunately altered the patient's prognosis, and this highlights the impact of this pandemic on our healthcare system. Finally, special considerations should also be made concerning COVID-19 transplanted patients [32-34]: indeed, immunosuppressive therapy might lead to an atypical clinical manifestation, such as unspecific viral disease or gastrointestinal symptoms [35]. This should be taken into account in order to minimize unexpected clinical complications and unpredictable outcomes.

In our institution, we have recently standardized the use of PPE to limit the possible transmission of disease during surgery. It has been speculated that laparoscopy might increase transmission due to the aerosolized biological fluids $[36,37]$. In line with this, we are now using extra protection (double gloves, shield, and filter mask) and filtered gas evacuation systems to avoid any possible transmission during minimally invasive procedures. However, as possible aerosolized fluids happen also as a result of cauterization during open surgery, the surgical community needs more evidence and guidelines concerning the proper use of equipment and resources to be used during surgery [38].

This study has some limitations mainly being the retrospective analysis that might have introduced selection bias. In this setting, anesthesiologists could have decided subjectively whether the patient required ICU admission or not. Furthermore, as mentioned above, our hospital is a national 
referral for the infectious diseases and we have already faced previous emergencies [17, 18]; therefore, the rapid adaptation that we have shown in this manuscript could be the result of experience and competences gained over years.

Since the recent worldwide spread of the disease, many editorials, letters, and expert opinions have been published on COVID-19 crisis and many have speculated that surgical activity has suffered the spread of this pandemic [12, 14, 23, 39-43]. To the best of our knowledge, this is the first manuscript presenting numbers and statistical analysis highlighting the changes in surgical practice during COVID19 outbreak. As we write, Italy is already in its fourth week of lockdown, and the emergency seems to have reached the peak. We will be probably moving toward more relaxed containment measures despite a total opening is unlikely. Meanwhile, countries worldwide are facing the ascending phase of this outbreak with a delay of days or even weeks. We believe it is important to share our anticipatory experience on how we have changed and adapted our surgical practice to face this crisis and optimized resources. Future evolution of this infection is uncertain: evidence, policies, and recommendations may change on daily bases hopefully improving the quality of care and minimizing the side effects on more patients. We must be prepared to face the consequent need for care in the coming months. By then, a progressive inverse trend should be encouraged, reallocating resources to assist those who suffered the most by the outbreak, both re-enforcing diagnostic steps, and treatment possibilities to counteract the delay we have so far accumulated.

\section{Conclusions}

Besides the emergency of patients infected by SARS-CoV-2 infection, our healthcare system is facing the side effects of COVID-19 pandemic. Adjustments were necessary in many environments as well as in the surgical field. A shift toward the exclusive management of oncologic diseases and urgent cases has been recently recommended worldwide. Rearrangements of resources require adaptations in clinical practice. Multidisciplinary meetings should be reworked in the context of COVID-19 reality and selection of patients should be enhanced to ensure cure and minimize loss of patients. We foresee a difficult phase for the time being in which all the non-COVID 19 health issues will re-emerge. International cooperation is required, and resources should be allocated accordingly to better tackle this future emergency.

\section{Compliance with ethical standards}

Conflict of interest All the authors declare no conflict of interest or financial support.
Research involving human participants and/or animals All procedures performed in studies involving human participants were in accordance with the ethical standards of the institutional and/or national research committee and with the 1964 Helsinki Declaration and its later amendments or comparable ethical standards.

Informed consent Informed consent was obtained from all individual participants involved in the study.

\section{References}

1. Wynants L, Van Calster B, Bonten MMJ, Collins GS, Debray TPA, De Vos M, Haller MC, Heinze G, Moons KGM, Riley RD, Schuit E, Smits LJM, Snell KIE, Steyerberg EW, Wallisch C, van Smeden M (2020) Prediction models for diagnosis and prognosis of covid-19 infection: systematic review and critical appraisal. BMJ 369:m1328

2. Liang W, Guan W, Chen R, Wang W, Li J, Xu K, Li C, Ai Q, Lu W, Liang H, Li S, He J (2020) Cancer patients in SARSCoV-2 infection: a nationwide analysis in China. Lancet Oncol 21(3):335-337

3. Wu Z, McGoogan JM (2020) Characteristics of and Important Lessons From the Coronavirus Disease 2019 (COVID-19) Outbreak in China: Summary of a Report of 72314 Cases From the Chinese Center for Disease Control and Prevention. JAMA 323:1239-1242

4. Dong E, Du H, Gardner L (2020) An interactive web-based dashboard to track COVID-19 in real time. Lancet Infect Dis 20:533-534

5. Elaborazione e gestione dati a cura del Dipartimento della Protezione Civile. Ministero della Salute. https://opendatadpc.maps. arcgis.com/apps/opsdashboard/index.html\#/b0c68bce2cce478 eaac82fe38d4138b1: COVID-19 Italia; 2020

6. Remuzzi A, Remuzzi G (2020) COVID-19 and Italy: what next? Lancet 395:1225-1228

7. Di Marzo F, Sartelli M, Cennamo R, Toccafondi G, Coccolini F, La Torre G, Tulli G, Lombardi M, Cardi M (2020) Recommendations for general surgery activities in a pandemic scenario (SARS-CoV-2). Br J Surg. https://doi.org/10.1002/bjs.11652

8. Guerci C, Maffioli A, Bondurri AA, Ferrario L, Lazzarin F, Danelli P (2020) COVID-19: How can a department of general surgery survive in a pandemic? Surgery 167:909-911

9. Patriti A, Eugeni E, Guerra F (2020) What happened to surgical emergencies in the era of COVID-19 outbreak? Considerations of surgeons working in an Italian COVID-19 red zone. Updates Surg. https://doi.org/10.1007/s13304-020-00779-6

10. Danese S, Cecconi M, Spinelli A (2020) Management of IBD during the COVID-19 outbreak: resetting clinical priorities. Nat Rev Gastroenterol Hepatol 17:253-255

11. Fiorino G, Allocca M, Furfaro F, Gilardi D, Zilli A, Radice S, Spinelli A, Danese S (2020) Inflammatory bowel disease care in the COVID-19 pandemic era: the Humanitas, Milan experience. J Crohns Colitis. https://doi.org/10.1093/ecco-jcc/jjaa058

12. Lisi G, Campanelli M, Spoletini D, Carlini M (2020) The possible impact of COVID-19 on colorectal surgery in Italy. Colorectal Dis. https://doi.org/10.1111/codi.15054

13. Ramirez PT, Chiva L, Eriksson AGZ, Frumovitz M, Fagotti A, Gonzalez Martin A, Jhingran A, Pareja R (2020) COVID-19 global pandemic: options for management of gynecologic cancers. Int J Gynecol Cancer 30(5):561-563

14. Iacobucci G (2020) Covid-19: all non-urgent elective surgery is suspended for at least three months in England. BMJ 368:m1106 
15. Lambertini M, Toss A, Passaro A, Criscitiello C, Cremolini C, Cardone C, Loupakis F, Viscardi G, Meattini I, Dieci MV, Ferrara R, Giusti R, Maio MD (2020) Cancer care during the spread of coronavirus disease 2019 (COVID-19) in Italy: young oncologists' perspective. ESMO Open 5(2):e000759

16. Nicastri E, Petrosillo N, Bartoli TA, Lepore L, Mondi A, Palmieri F, D’Offizi G, Marchioni L, Murachelli S, Ippolito G, Antinori A (2020) National Institute for the Infectious Diseases "L. Spallanzani", IRCCS. Recommendations for COVID-19 clinical management. Infect Dis Rep 12(1):8543

17. Nicastri E, Castilletti C, Biava M, Fusco FM, Petrosillo N, Puro V, Lauria FN, Capobianchi MR, Di Caro A, Ippolito G (2017) Enabling rapid response to the 2014-2016 ebola epidemic: the experience and the results of the National Institute for Infectious Diseases Lazzaro Spallanzani. Adv Exp Med Biol 972:103-122

18. Nicastri E, Petrosillo N, Macri G, Ippolito G (2003) Severe acute respiratory syndrome: the first transmissible disease of the $21 \mathrm{st}$ century. Recenti Prog Med 94(7-8):295-303

19. Charlson ME, Pompei P, Ales KL, MacKenzie CR (1987) A new method of classifying prognostic comorbidity in longitudinal studies: development and validation. J Chronic Dis 40(5):373-383

20. Goldman L, Caldera DL, Nussbaum SR, Southwick FS, Krogstad D, Murray B, Burke DS, O’Malley TA, Goroll AH, Caplan CH, Nolan J, Carabello B, Slater EE (1977) Multifactorial index of cardiac risk in noncardiac surgical procedures. N Engl J Med 297(16):845-850

21. Mayhew D, Mendonca V, Murthy BVS (2019) A review of ASA physical status - historical perspectives and modern developments. Anaesthesia 74(3):373-379

22. Strasberg SM (2005) Nomenclature of hepatic anatomy and resections: a review of the Brisbane 2000 system. J Hepatobiliary Pancreat Surg 12(5):351-355

23. Kiran T, Saket G (2020) Are we harming cancer patients by delaying their cancer surgery during the COVID-19 pandemic? Ann Surg (In press)

24. Bartlett DL, Howe JR, Chang G, Crago A, Hogg M, Karakousis G, Levine E, Maker A, Mamounas E, McGuire K, Merchant N, Shibata D, Sohn V, Solorzano C, Turaga K, White R, Yang A, Yoon S, Society of Surgical O (2020) Management of cancer surgery cases during the COVID-19 pandemic: considerations. Ann Surg Oncol 27:1717-1720

25. Desai A, Sachdeva S, Parekh T, Desai R (2020) COVID-19 and cancer: lessons from a pooled meta-analysis. JCO Glob Oncol 6:557-559

26. Berardi G, Levi Sandri GB, Colasanti M, Ettorre GM (2020) Readaptation of surgical practice during COVID-19 outbreak: what has been done, what is missing and what to expect. Br J Surg. https://doi.org/10.1002/bjs.11698

27. Mazzaferro V, Danelli P, Torzilli G, Busset MDD, Virdis M, Sposito C (2020) A combined approach to priorities of surgical oncology during the COVID-19 epidemic. Ann Surg. https://doi. org/10.1097/SLA.0000000000004005

28. Mercantini P, Lucarini A, Mazzuca F, Osti MF, Laghi A (2020) How technology can help in oncologic patient management during COVID-19 outbreak. Eur J Surg Oncol. https://doi.org/10.1016/j. ejso.2020.04.050

29. Ueda M, Martins R, Hendrie PC, McDonnell T, Crews JR, Wong TL, McCreery B, Jagels B, Crane A, Byrd DR, Pergam SA, Davidson NE, Liu C, Stewart FM (2020) Managing cancer care during the COVID-19 pandemic: agility and collaboration toward a common goal. J Natl Compr Canc Netw. https://doi.org/10.6004/ jncen. 2020.7560
30. Pellino G, Spinelli A (2020) How COVID-19 outbreak is impacting colorectal cancer patients in Italy: a long shadow beyond infection. Dis Colon Rectum. https://doi.org/10.1097/DCR.00000 00000001685

31. Zheng MH, Boni L, Fingerhut A (2020) Minimally invasive surgery and the novel coronavirus outbreak: lessons learned in China and Italy. Ann Surg. https://doi.org/10.1097/SLA.0000000000 003924

32. Agnes S, Andorno E, Avolio AW, Baccarani U, Carraro A, Cescon M, Cillo U, Colledan M, De Carlis L, De Simone P, De Ville De Goyet J, Di Benedetto F, Ettorre GM, Gringeri E, Gruttadauria S, Lupo LG, Mazzaferro V, Regalia E, Romagnoli R, Rossi GE, Rossi M, Spada M, Tisone G, Vennarecci G, Vivarelli M, Zamboni F, Boggi U (2020) Preliminary analysis of the impact of COVID-19 outbreak on Italian liver transplant programs. Liver Transpl. https://doi.org/10.1002/lt.25790

33. Angelico R, Trapani S, Manzia TM, Lombardini L, Tisone G, Cardillo M (2020) The COVID-19 outbreak in Italy: initial implications for organ transplantation programs. Am J Transplant. https ://doi.org/10.1111/ajt.15904

34. Lauterio A, De Carlis R, Belli L, Fumagalli R, De Carlis L (2020) How to guarantee liver transplantation in the north of Italy during the COVID-19 pandemic. A sound transplant protection strategy. Transpl Int. https://doi.org/10.1111/tri.13633

35. Guillen E, Pineiro GJ, Revuelta I, Rodriguez D, Bodro M, Moreno A, Campistol JM, Diekmann F, Ventura-Aguiar P (2020) Case report of COVID-19 in a kidney transplant recipient: Does immunosuppression alter the clinical presentation? Am J Transplant. https://doi.org/10.1111/ajt.15874

36. Li CI, Pai JY, Chen CH (2020) Characterization of smoke generated during the use of surgical knife in laparotomy surgeries. J Air Waste Manag Assoc 70(3):324-332

37. Spinelli A, Pellino G (2020) COVID-19 pandemic: perspectives on an unfolding crisis. Br J Surg 107(7):785-787

38. Rohan P, O'Reilly MK, Gibney B, Nason GJ (2020) Supply of personal protective equipment (PPE) during the Covid-19 pandemic. Ir Med J 113(4):66

39. Aminian A, Safari S, Razeghian-Jahromi A, Ghorbani M, Delaney CP (2020) COVID-19 outbreak and surgical practice: unexpected fatality in perioperative period. Ann Surg. https://doi.org/10.1097/ SLA.0000000000003925

40. Kurihara H, Bisagni P, Faccincani R, Zago M (2020) COVID-19 outbreak in Northern Italy: viewpoint of the Milan area surgical community. J Trauma Acute Care Surg 88(6):719-724

41. McBride KE, Brown K, Fisher OM, Steffens D, Yeo D, Koh CE (2020) Impact of the COVID-19 pandemic on surgical services: early experiences at a nominated COVID-19 centre. ANZ J Surg 90(5):663-665

42. Peloso A, Moeckli B, Oldani G, Triponez F, Toso C (2020) Response of a European surgical department to the COVID-19 crisis. Swiss Med Wkly 150:w20241

43. Zoia C, Bongetta D, Veiceschi P, Cenzato M, Di Meco F, Locatelli D, Boeris D, Fontanella MM (2020) Neurosurgery during the COVID-19 pandemic: update from Lombardy, northern Italy. Acta Neurochir (Wien). https://doi.org/10.1007/s00701-020-04305-w

Publisher's Note Springer Nature remains neutral with regard to jurisdictional claims in published maps and institutional affiliations. 